\title{
Produtos da reação de Maillard em alimentos: implicações para a saúde
}

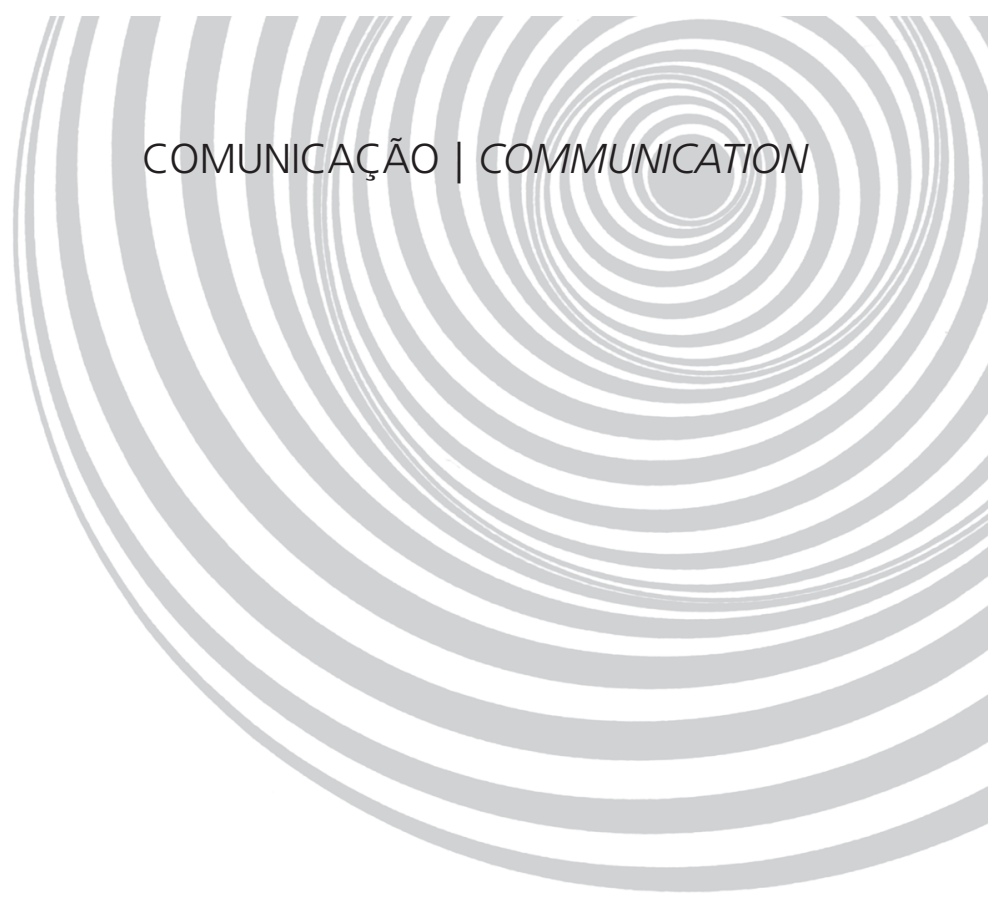

\author{
Maillard reaction products in foods: \\ implications for human health
}

Julianna SHIBAO'

Deborah Helena Markowicz BASTOS ${ }^{1}$

RE S U M O

A reação de Maillard é uma reação de escurecimento não enzimático que pode ocorrer em alimentos e em organismos vivos. Esta revisão tem como objetivo analisar a formação e o papel dos produtos originados a partir da reação de Maillard e seus efeitos na saúde. Para isso foram realizados levantamentos bibliográficos nas bases da área, sem restrição de data. Os resultados da revisão apontam que produtos carbonílicos intermediários da reação de Maillard e da peroxidação reagem facilmente com grupamentos aminas de proteínas e ácidos nucleicos, levando a modificações biológicas que podem resultar em complicações observadas no diabetes, aterosclerose e doenças neurodegenerativas. O consumo de produtos da reação de Maillard aumentou nas últimas décadas, devido ao aumento do consumo de alimentos industrializados que, em geral, sofreram processamento térmico. Essas substâncias são biodisponíveis em alguma proporção e, embora ainda não haja consenso sobre os possíveis efeitos deletérios à saúde decorrentes de sua ingestão, a comunidade científica tem expressado preocupação com as implicações em processos patológicos de que participam. Diante desses achados, ressalta-se a necessidade de estimar o consumo dos produtos da reação de Maillard, principalmente por populações vulneráveis, como crianças e diabéticos, a fim de, se necessário, estabelecer consumos diários aceitáveis e ampliar o conhecimento com vistas ao estabelecimento, no futuro, de limites para a indústria de alimentos.

Termos de indexação: Análise de alimentos. Consumo de alimentos. Glicação. Nutrição. Reação de Maillard.

A B S T R A C T

Maillard reaction is the nonenzymatic browning that occurs in foods and living organisms. The objective of this review is to analyze the formation and role of Maillard reaction products and their effects on human health. A literature search was done in the relevant databases for all articles published on the subject. The results of the review show that intermediate carbonyl compounds of the Maillard reaction and peroxidation easily react with the amino groups of proteins and nucleic acids leading to biological changes that can, in turn, lead to

\footnotetext{
1 Universidade de São Paulo, Faculdade de Saúde Pública, Departamento de Nutrição. Av. Dr. Arnaldo, 715, Cerqueira César, 01246-904, São Paulo, SP, Brasil. Correspondência para/Correspondence to D.H.M. BASTOS. E-mail: <dmbastos@usp.br>.
} 
896 | J. SHIBAO \& D.H.M. BASTOS

complications, such as those seen in diabetes, atherosclerosis and degenerative diseases. Consumption of Maillard reaction products increased in the last decades because of the increased consumption of processed foods, since the production of many processed foods may require the use of heat. These substances are bioavailable to some degree and, although there is no consensus about their harmful effects on human health, the scientific community has expressed concern with their implications on the pathological processes of which they are part. These findings suggest that the intake of these substances, especially by vulnerable individuals, such as children and diabetics, should be estimated for the establishment of acceptable daily intakes, if necessary. More knowledge about these substances may also result in the establishment of a maximum MRP level in processed foods.

Indexing terms: Food analysis. Food consumption. Glycation. Nutrition. Maillard reaction.

\section{N T R O D U Ç Ã O}

Nas últimas décadas, os hábitos alimentares da população brasileira mudaram, e observa-se, com preocupação, o aumento do consumo das refeições feitas fora de casa, em sistemas do tipo fast food, e de alimentos industrializados ${ }^{1}$.

Antes de consumidos os alimentos, a maior parte deles sofre processamento térmico, o que garante a segurança microbiológica, a inativação de algumas enzimas, a degradação de substâncias tóxicas e, ainda, o desenvolvimento de substâncias responsáveis pelo aroma, cor e sabor, melhorando a sua palatabilidade 2,3. Essas substâncias (compostos denominados genericamente de Produtos da Reação de Maillard - PRM) são características da Reação de Maillard (RM), que ocorre durante o processamento térmico e/ou armazenamento prolongado de alimentos que contêm proteínas e açúcares redutores ${ }^{3,4}$. Esses compostos são biologicamente ativos e podem resultar em benéficos à saúde, por apresentarem atividade antioxidante e antimutagênica. Por outro lado, porém, o consumo de PRM pode interferir em processos nutricionais importantes, como diminuir a biodisponibilidade de minerais e o valor biológico de proteínas, pelo comprometimento, na reação, de resíduos de aminoácidos essenciais, com consequentes alterações da estrutura proteica ou, ainda, inibição de enzimas digestivas ${ }^{4,5}$.

Esta revisão tem como objetivo analisar as informações disponíveis na literatura sobre a reação de Maillard e sua relação com a saúde, dado tratar-se de tema controverso e atual. Adotaram-se, para a consulta às bases de dados, os seguintes descritores: Maillard reaction, Advanced Glycation Endproducts, glycation e glycotoxins, agrupados de maneiras diversas para aperfeiçoar a busca. Os artigos de revisão e os artigos originais pesquisados compreendiam aqueles, na língua inglesa, portuguesa e espanhola, que tratam do mecanismo e fatores que interferem na formação dos PRM e sua relação com a saúde.

\section{REAÇÃO DE MAILLARD}

O fenômeno de que os alimentos escurecem à medida que são aquecidos é provavelmente conhecido desde a descoberta do fogo, há mais de 300 mil anos. As reações químicas que resultam nesse fenômeno foram primeiramente descritas em 1912 pelo bioquímico francês Louis-Camille Maillard, que publicou o primeiro estudo sistemático mostrando que aminoácidos e açúcares redutores iniciam uma complexa cascata de reações durante o aquecimento, resultando na formação final de substâncias marrons chamadas de melanoidinas ${ }^{2,3}$.

A RM inicia-se com o ataque nucleofílico do grupo $\alpha$-carbonílico de um açúcar redutor, por exemplo, ao grupamento amina de proteínas ${ }^{2-4}$. A ocorrência da reação em alimentos depende de vários fatores: temperaturas elevadas (acima de $40^{\circ} \mathrm{C}$ ), atividade de água na faixa de 0,4 a 0,7, $\mathrm{pH}$ na faixa de 6 a 8 (preferencialmente alcalino), umidade relativa de $30 \%$ a $70 \%$, presença de íons metálicos de transição como $\mathrm{Cu}^{2+}$ e $\mathrm{Fe}^{2+}$, que podem catalisar a reação ${ }^{6,7}$.

Além desses fatores, a composição do alimento também influência na ocorrência da RM. 
O tipo de açúcar redutor interfere na velocidade de reação com os grupamentos amina, sendo o açúcar redutor mais reativo a xilose, seguida de arabinose, glicose, maltose e frutose, indicando que as pentoses são mais reativas do que as hexoses. Ainda, os açúcares redutores diferem na via de escurecimento, sendo que as cetoses são mais reativas para a formação de produtos de Heyns, enquanto as aldoses o são para a formação de produtos de Amadori. Além dos açúcares, os tipos de aminoácidos também interferem na velocidade de reação. A lisina é cerca de 2 a 3 vezes mais reativa quando comparada aos outros aminoácidos, devido à presença de grupamentos $\alpha \mathrm{e}$ $\varepsilon$-amino em sua estrutura. Na sequência, os aminoácidos básicos e não polares (arginina, fenilalanina, leucina, isoleucina e valina) são os mais reativos, seguidos dos aminoácios ácidos (ácido glutâmico e ácido aspártico). A cisteína, por ser um aminoácido sulfurado, é menos reativa ${ }^{5-8}$.

Didaticamente, a RM é dividida em três fases (inicial, intermediária e final), conforme o esquema inicialmente proposto por Hodge em 1953 e citado por Nursten 7,9 . Alguns dos compostos formados são identificados como característicos de cada uma dessas fases, que, no entanto, não ocorrem de forma sequencial, mas em cascata.

No estágio inicial ocorre a condensação da carbonila de um açúcar redutor, por exemplo, com um grupamento amina proveniente de aminoácidos livres ou de proteínas, levando à formação de glicosil/frutosilaminas $\mathrm{N}$-substituídas. Esse é o primeiro produto estável formado da RM (produto de Amadori). Em alimentos que contenham proteína, o grupamento amino do resíduo de lisina é o alvo principal para o ataque de açúcares redutores. Os produtos formados nessa etapa não possuem cor, fluorescência ou absorção característica na região do ultravioleta ${ }^{8-10}$. Na etapa seguinte, prolongando-se o aquecimento ou armazenamento, os produtos de Amadori dão origem a uma série de reações (desidratação, enolização e retroaldolização), resultando em compostos dicarbonílicos, redutonas e derivados do furfural, ou ainda em produtos da degradação de Strecker (produtos de degradação de aminoácidos). Nesta fase, é observado o aumento da geração produtos fluorescentes e de substâncias capazes de absorver radiação na região do ultravioleta9,10.

No último estágio da RM, os produtos intermediários (dicarbonílicos), muito reativos, podem reagir com, por exemplo, resíduos de lisina ou arginina em proteínas, formando compostos estáveis. Nessa fase ocorrem reações de fragmentação e polimerização, com a geração de melanoidinas (compostos de coloração marrom e alto peso molecular) e de compostos fluorescentes.

Ao longo do processo são formados compostos voláteis, tais como cetonas e aldeídos, que conferem o aroma característico aos produtos termicamente processados ${ }^{7-11}$.

A reação de Maillard ocorre também nos organismos vivos, sendo, nesse caso, denominada "glicação" 12-14. A identificação da hemoglobina glicada em pacientes diabéticos foi o marco para os estudos desse processo no organismo e suas implicações para a saúde. A reação pode ocorrer, in vivo, pela via do estresse carbonílico, na qual a oxidação de lipídeos ou de açúcares gera compostos dicarbonílicos intermediários altamente reativos ${ }^{12-14}$. A glicólise e a autoxidação de glicose, por exemplo, produzem metilglioxal e glioxal, os quais interagem com aminoácidos para formar produtos finais dessa reação. Esses compostos dicarbonílicos chegam a ser 20 mil vezes mais reativos do que a glicose e estão presentes tanto in vivo quanto nos alimentos. A velocidade da glicação é maior em estados de hiperglicemia, em que a glicose se liga a proteínas de tecidos independentes de insulina e de longa vida ${ }^{4,13,14}$.

Assim como a RM em alimentos, a glicação in vivo também pode ser dividida em estágios cinéticos. A primeira fase, chamada de estressores, é composta de agentes carbonílicos que podem dar início à reação. A segunda fase, chamada de propagadores, é formada de outros compostos carbonílicas reativas proveniente dos estressores anteriores. A última fase é a dos produ- 
898 | J. SHIBAO \& D.H.M. BASTOS

tos finais, que ativam o processo de envelhecimento celular resultante da glicação ${ }^{12,14,15}$.

A RM nos alimentos confere e influencia atributos sensoriais fundamentais para a aceitação de alimentos termicamente processados, devido à geração de compostos voláteis, responsáveis pelo aroma e sabor (aldeídos e cetonas), bem como pela cor (melanoidinas) e textura. Por outro lado, pode originar compostos que são adversos a saúde humana, como a acroleína e as aminas heterocíclicas aromáticas ${ }^{16,17}$.

\section{Produtos da Reação de Maillard e produtos de glicação avançada}

Os Produtos da Reação de Maillard correspondem a um grupo heterogêneo de compostos químicos, com ampla variação no peso molecular, formados em alimentos e em sistemas biológicos $^{12,15,17}$.

Compostos representativos representativas dessa reação, encontrados em alimentos e em sistemas biológicos são: carboximetilisina, hidroximetilfurfural, pentosidina, carboxietilisina, pirralina, vesperlisina A, dímero de glioxal-lisina, dímero metilglioxal-lisina, glicosepana ${ }^{12,15,18}$.

Os PRM estão presentes em alimentos submetidos a qualquer tipo de tratamento térmi$\mathrm{co}$, incluindo alimentos fritos, assados em churrasqueiras, cozidos em forno convencional ou de micro-ondas, sendo a temperatura o parâmetro crítico relativamente a essa reação. Assim, métodos mais brandos de cozimento e com alta atividade de água, como preparações ensopadas e a vapor, geram teores menores de PRM. O teor de PRM em peito de frango, por exemplo, pode variar de $1.100 \mathrm{kU} / 100 \mathrm{~g}$ - quando cozido em cocção úmida - a $4.700 \mathrm{kU} / 100 \mathrm{~g}$, quando frito (unidades referentes a CML dosados por método de ELISA). A preparação frita atinge cerca de um terço da recomendação de ingestão desses compostos por diabéticos, a qual não deve ser ultrapassar 16.000kU por dia 17,19,20.

Os PRM têm sido analisados em diferentes alimentos, com a finalidade de produzir um banco de dados sobre o teor dessas substâncias em diferentes preparações, para estimar quantidades ingeridas e a ingestão máxima recomendada. Para a quantificação dos PRM e para a avaliação da intensidade do tratamento térmico e consequente perda de valor nutricional de alimentos, têm sido empregados marcadores como a intensidade de fluorescência e teor de furosina, hidroximetilfurfural, carboximetilisina, pentosidina, pronilisina, pirralina. As técnicas analíticas mais comumente utilizadas compreendem a espectrofotometria, a cromatografia líquida e gasosa de alta eficiência e os métodos imunológicos (ELISA) $)^{17,20}$.

A Tabela 1 sumariza os dados de teor de PRM encontrados em alimentos, publicados na literatura internacional. Os alimentos presentes no grupo "gorduras" apresentam teores maiores de PRM, devido ao favorecimento de reações entre as aminas e os produtos da oxidação lipídica. Nesse grupo estão incluídos manteiga, requeijão, margarina, maionese, óleos e oleaginosas. Altos teores de PRM também são encontrados em alimentos com alto teor de proteínas, como carnes, peixes e ovos. Os alimentos ricos em carboidratos contêm teores intermediários de PRM e merecem atenção, tanto por sua participação na dieta, quanto por ser a lisina o aminoácido limitante em cereais. Nesse grupo estão incluídos panquecas, pães, frutas e algumas verduras ${ }^{17,21}$.

Como e quais PRM e/ou seus metabólitos são absorvidos e metabolizados é ainda um tema controverso na literatura. Estudos com ratos indicam que $20 \%$ dos PRM são absorvidos pelo trato gastrointestinal, por difusão passiva. Destes, 30\% são excretados pelos rins e cerca de $3 \%$ pelas fezes. Cerca de $70 \%$ dos PRM metabolizados acumulam-se em vários tecidos, como pâncreas, fígado e, principalmente, rins; já os PRM que não foram absorvidos são degradados pelas bactérias no intestino ou eliminados. Enquanto há evidências de que altas concentrações de PRM no intestino aumentam a concentração local de nitrogênio, favorecendo o desenvolvimento de doenças intestinais como colite, alguns PRM, por outro lado, apresentam efeito prebiótico, indicando que 
Tabela 1. Teor de Produtos da Reação de Maillard (PRM) em alguns alimentos.

\begin{tabular}{|c|c|c|c|}
\hline Alimento & Conteúdo de PRM* (kU/100g) & Alimento & Conteúdo de PRM* $(\mathrm{kU} / 100 \mathrm{~g})$ \\
\hline \multicolumn{4}{|l|}{ Grupo dos óleos } \\
\hline Manteiga & 26480 & Molho para salada & 740 \\
\hline Margarina & 17520 & Semente de abóbora & 1853 \\
\hline Maionese & 9400 & Pistache & 380 \\
\hline Óleo de oliva & 11900 & Cream cheese & 10883 \\
\hline Amendoim & 6447 & Amêndoa & 5650 \\
\hline \multicolumn{4}{|l|}{ Grupo das proteínas } \\
\hline Carne bovina grelhada & 7479 & Ovos fritos & 2749 \\
\hline Carne bovina cozida & 2687 & Bacon & 91577 \\
\hline Carne de frango grelhada & 6639 & Atum enlatado com óleo & 5113 \\
\hline Carne de frango cozida & 1210 & Queijo parmesão gratinado & 16900 \\
\hline Peixe assado & 1212 & Queijo Cheddar & 5523 \\
\hline Peixe frito & 3083 & Linguiça de porco & 5943 \\
\hline Ovos cozidos & 90 & Camarão frito & 4328 \\
\hline \multicolumn{4}{|l|}{ Grupo dos carboidratos } \\
\hline Panquecas & 2263 & Croissant & 1113 \\
\hline Pães & 133 & Granola & 427 \\
\hline Biscoitos & 1647 & Waffle & 2870 \\
\hline Flocos de milho & 233 & Macarrão & 242 \\
\hline
\end{tabular}

*PRM: referente a carboximetilisina determinada por ensaio imunoenzimático (ELISA).

Adaptado de Vlassara et al. ${ }^{42}$

o tema é controverso e pouco se conhece sobre a biotransformação desses compostos pela microbiota intestinal22-25.

Nos últimos 50 anos o consumo desses produtos aumentou cerca de cinquenta vezes na dieta ocidental 24,25 , em especial na alimentação de adolescentes ${ }^{26}$, o que tem levado diversos autores a sugerir a necessidade de estabelecer limites de ingestão, ou, ainda, utilizar métodos culinários que reconhecidamente minimizem a formação desses compostos 14,20,27,28.

\section{Efeitos biológicos dos produtos da reação de Maillard ou da reação de glicação avançada}

O primeiro trabalho indicando que PRM poderiam ser prejudiciais a processos biológicos foi publicado em 1949. Nesse trabalho, observou-se que PRM presentes no melaço de cana inibiam a fermentação e, consequentemente, a produção de álcool. Na sequência, foram avaliados os efeitos fisiológicos desses compostos na inibição do cres- cimento celular, redução da digestibilidade e absorção de proteínas, hipertrofia de órgãos, mutações celulares, redução das atividades de enzimas pancreáticas, intestinais, hepáticas e formação de complexos com metais ${ }^{2,3}$.

Há consenso sobre o efeito deletério da RM quanto ao comprometimento de aminoácidos essenciais, notadamente a lisina, levando à perda do valor biológico de alimentos. O processamento térmico de cereais matinais leva à perda de $20 \%$ a $30 \%$ da lisina e à inibição da atividade de enzimas digestivas ${ }^{28}$. Como a lisina é um aminoácido limitante em produtos à base de cereais, as condições de manufatura devem ser escolhidas cuidadosamente, visando a garantir não apenas as características sensoriais, mas também o valor nutricional adequado 13,26,29.

Os Produtos de Glicação Avançada (AGE), por sua vez, são implicados no envelhecimento e na perda de funcionalidade de tecidos que não são dependentes de insulina e, por isso, encontram-se expostos a concentrações elevadas de glicose (como o cristalino, a membrana basal das arteríolas, as células nervosas e os tecidos intersti- 
ciais da pele). A catarata e aterosclerose, em alguma extensão, são patologias resultantes da glicação entre a glicose sanguínea com proteínas de longa duração presentes nesses tecidos ${ }^{20,30}$.

Os AGE podem causar danos aos tecidos por: (1) modificar a função da proteína devido a alterações em sua estrutura/configuração; (2) modificar o tecido em si, devido às ligações cruzadas (cross-links) inter e intramolecular; (3) favorecer a formação de radicais livres; e (4) induzir resposta inflamatória após ligarem-se a receptores específicos RAGE (Receptor de Produtos da Glicação Avançada), receptores da membrana celular, cuja atividade aumenta proporcionalmente à concentração de $A G E$ presentes. A interação AGE-RAGE modula vias pró-inflamatórias com ativação transcricional e expressão alterada de vários mediadores inflamatórios, como as citocinas, desencadeando reações inflamatórias (Figura 1$)^{31-33}$.

A ligação de AGE a tecidos como a retina, endotélio, mesênquima e células renais, favorece

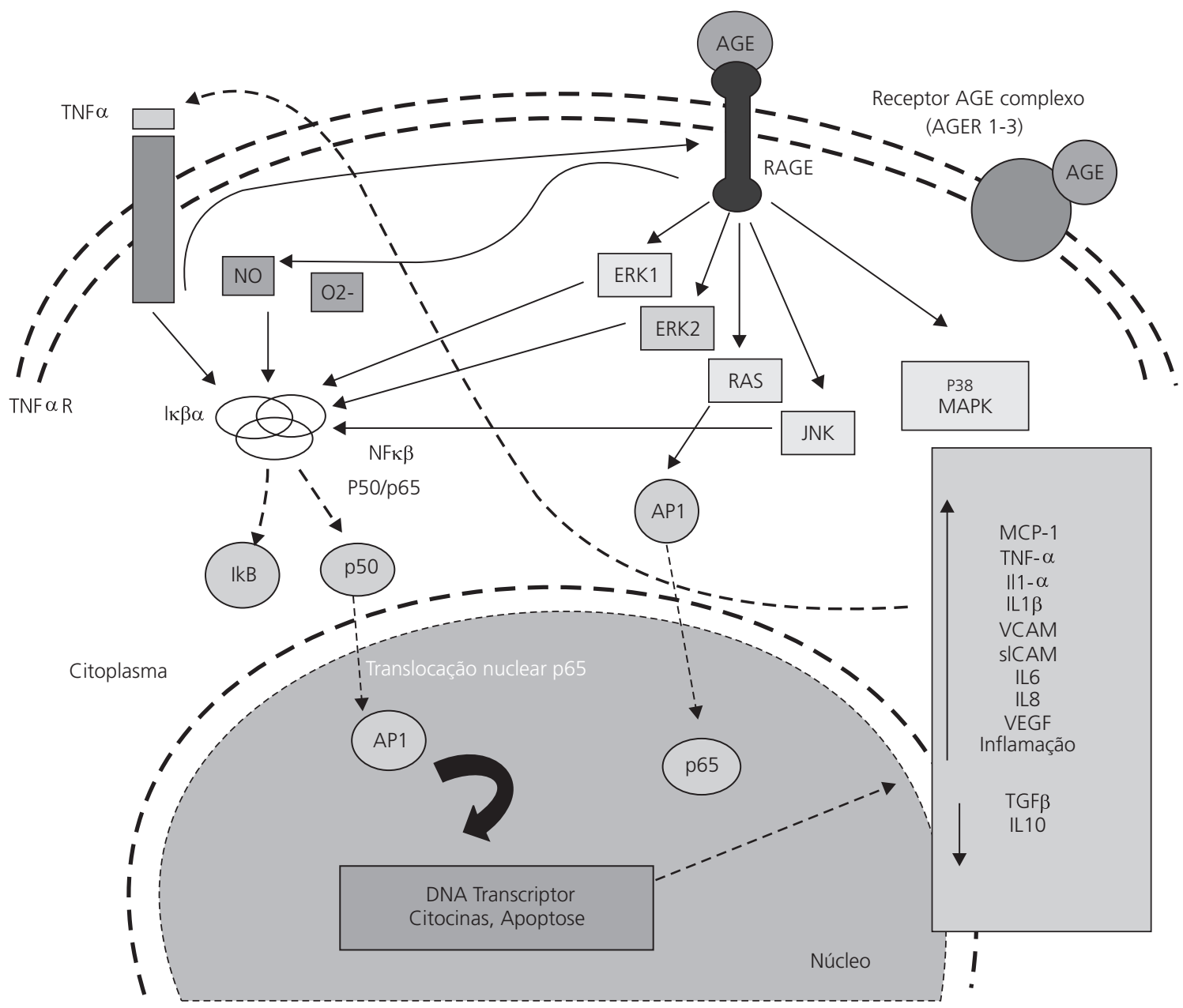

Figura 1. Diagrama exemplificando o envolvimento de RAGE (receptores de AGE) em processos inflamatórios e aterogênicos.

*adaptada de Glenn \& Stitt23.

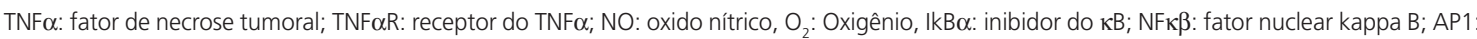
proteína ativadora 1; JNK: jun n-terminal quinase; MAPK: proteína quinase mitógeno-ativada; MCP-1: proteína quimioatratora de monócitos 1; IL1 $\alpha$ : Interleucina 1 Alfa; IL1ß: interleucina 1 beta; IL-6: Interleucina 6; IL-8: Interleucina 8; IL -10: interleucina 10; VCAM: molécula de adesão vascular 1; sICAM-1: molecula intracelular de adesão-1; VEGF: fator de crescimento endotelial vascular, TGF- $\beta$ : fator de crescimento transformador beta. 
a disfunção endotelial (prejudicando a vasodilatação devido à diminuição da produção de monóxido de nitrogênio - NO), a ativação acelerada de macrófagos para as células espumosas, a diminuição da flexibilidade das células musculares lisas, comprometendo a complacência arterial, e torna a fração LDL-colesterol mais susceptível à oxidação ${ }^{31,33}$ (Figura 1). Os AGE estão associados a complicações renais, disfunções endoteliais e patologias neurodegenerativas, processo em que se observa elevada concentração desses compostos, o que possivelmente se relaciona à disfunção neurona ${ }^{34} \mathrm{e}$, ainda, a fatores de risco do desenvolvimento de doenças cardiovasculares 32,34-39.

Indivíduos diabéticos, em especial, apresentam diversas complicações devidas aos processos de glicação; atualmente, recomenda-se que sua dieta seja controlada quanto aos teores de PRM. Apesar de não haver consenso sobre os limites de ingestão segura, a redução de 50\% na ingestão de PRM na dieta de indivíduos diabéticos resultou em decréscimo de 30\% dos teores plasmáticos desses mesmos compostos, em apenas um mês de intervenção. Observou-se também redução do peso corporal e melhora do prognóstico da doença, embora o nível de hemoglobina glicosilada -HbA1c- não tenha sido modifica$\mathrm{do}^{39-41}$.

Estima-se que os indivíduos que consomem maiores proporções de PRM estejam expostos a maior risco de desenvolver complicações do diabetes, como disfunções vasculares e renais ${ }^{20,41,43}$, e que o consumo seguro para eles seja de, no máximo, 16 mil unidades de PRM por dia (medida relativa à carboximetilisina) ${ }^{32,35}$.

Estudos com ratos mostraram que os animais que receberam dietas com altos teores de PRM por 6 meses desenvolveram diabetes mellitus tipo II, enquanto os animais do grupo-controle (dieta equivalente em composição e valor energético, mas com teores de PRM reduzidos) não só não desenvolveram o diabetes, como também apresentaram menores níveis de glicemia ${ }^{44}$.

O consumo de PRM está positivamente associado à síntese de citosinas inflamatórias (TNF- $\alpha$ e proteína $C$ reativa) e ao desenvolvimento de nefropatologias, conforme demonstrado em estudos com animais ${ }^{38,43,45}$. Dessa forma, identificar os níveis de PRM em alimentos produzidos e comercializados para pessoas com diabetes e deficiência renal, pode ser uma estratégia que viabiliza a orientação dessa população, contribuindo para a diminuição do risco de desenvolvimento de patologias decorrentes dessa condição de saúde ${ }^{41,43,45}$.

No entanto, há que enfatizar que o tema é bastante controverso, dadas as evidências de que algumas substâncias desse grupo, principalmente as melanoidinas, apresentam atividade antimutagênica, antioxidante e anticariogênica, entre outras, e, em alguma extensão, protegem o organismo. A seguir, o Quadro 1 apresenta um resumo das principais atividades biológicas benéficas desses compostos.

Quadro 1. Possíveis ações benéficas dos PRM e melanoidinas em sistemas modelo*.

\begin{tabular}{ll}
\hline Ação & Principais resultados obtidos \\
\hline Prebiotico e quimioprevenção (no intestino) & $\begin{array}{l}\text { Melanoidinas estimulam o crescimento de bactérias benéficas no intestino e dimi- } \\
\text { nuem a biodisponibilidade de aminas heterocíclicas. }\end{array}$ \\
Quimiopreventiva & $\begin{array}{l}\text { Indução de enzimas quimiopreventivas (enzimas com a propriedade de suprimir ou } \\
\text { prevenir a progressão carcinogênica para carcinoma invasor) in vitro e in vivo. }\end{array}$ \\
Capacidade antioxidante & Inibição da peroxidação lipídica em hepatócitos isolados. Aumento da capacidade \\
& antioxidante no plasma de humanos após administração de alimentos contendo \\
& melanoidinas. \\
Mutagenicidade e genotoxicidade & Melanoidinas apresentam efeitos mutagênicos pouco pronunciados in vitro. \\
\hline
\end{tabular}

*Adaptado de Somoza ${ }^{6}$ 


\section{CONSIDERAÇÕ ES FINAIS}

A ingestão de substâncias formadas pela reação de Maillard na dieta ocidental aumentou consideravelmente nas últimas décadas, em função do incremento do consumo de alimentos termicamente processados. Esse quadro pode acarretar desequilíbrio relativamente à reação de glicação, com importantes implicações para a população com diabetes e insuficiência renal. Pode, ainda, aumentar o risco de doenças vasculares e neurodegenerativas em grupos populacionais que estejam expostos ao consumo excessivo dessas substâncias.

Os PRM que são normalmente avaliados como parte integrante das dietas atuais, como a carboximetillisina, o hidroximetilfurfural e a pentosidina, não representam a totalidade dos produtos formados em alimentos durante o aquecimento, mas são marcadores da intensidade do tratamento térmico e podem ser determinados com exatidão/precisão nos laboratórios de análise de alimentos. O efeito dos produtos da reação de Maillard, presentes nos alimentos processados, é um tema aberto à discussão, na medida em que tais substâncias diminuem a biodisponibilidade de minerais e participam da etiologia de processos biológicos deletérios. A comunidade científica precisa avaliar se o grau de exposição da população a tais produtos pode se tornar um problema de saúde pública, o que, certamente, exigirá ações por parte das indústrias de alimen-tos, a fim de minimizar os efeitos dos PRM formados, sem perda da qualidade sensorial.

\section{A GRADECIMENTOS}

Ao Instituto Ajinomoto, pela bolsa à autora J. SHIBAO, à Fundação de Amparo à Pesquisa do Estado de São Paulo, pelo auxílio financeiro (processo 2008/03744-2); e ao Conselho Nacional de Desenvolvimento Científico e Tecnológico, pela bolsa à autora Bastos.

\section{COLABORADORES}

J. SHIBAO responsável pela laboração do projeto de pesquisa, busca bibliográfica, redação inicial, discussão dos resultados e redação do artigo. D.H.M. BASTOS responsável pela concepção do trabalho, participação na elaboração do projeto de pesquisa, na discussão dos resultados e na redação do artigo.

\section{REFERÊ NCIAS}

1. Instituto Brasileiro de Geografia e Estatística. Pesquisa de orçamentos familiares, 2002-2003. Aquisição alimentar domiciliar per capita, Brasil e grandes regiões. Rio de Janeiro: IBGE; 2004.

2. Finot PA. Historical perspective of the Maillard reaction in food science. Ann NY Acad Sci. 2005; 1043:1-8.

3. Friedman M. Food browning and its prevention: an overview. J Agric Food Chem. 1996; 44:631-53.

4. Nunes CS, Baptista AO. Implicações da reação de Maillard nos alimentos e nos sistemas biológicos. Rev Port Ciênc Vet. 2001; 96(538):53-9.

5. Morales FJ, van Boekel MAJS. A study on advanced Maillard reaction in heated casein sugar solutions. Int Dairy J. 1997; 7:675-83.

6. Kwak EJ, Lim SI. The effect of sugar, amino acid, metal íon and $\mathrm{NaCl}$ on model Maillard reaction under pH control. Amino Acids. 2004; 27:85-90. doi: 10.1007/s00726-004-0067-7.

7. Hodge JE. Chemistry of browning reactions in models systems. J Agric. Food Chem. 1953; 1(15): 928-43.

8. Martins SIFS, Jongen WMF, Boekel MAJSV. A review of Maillard reaction in food and implications to kinects modelling. Trends Food Sci Technol. 2000; 11(9-10):364-73.

9. Nursten H. The Maillard Reaction: Chemistry, biochemistry and Implications. Cambridge: The Royal Society of Chemistry; 2005.

10. Delgado-Andrade C, Rufian-Henares JA, Morales FJ. Colour and fluorescence measurement as unspecific markers for the Maillard reaction. In: Delgado-Andrade C, Rufian-Henares JA. Assessing the generation and bioactivity of neo-formed compounds in thermally treated foods. Granada: Atrio; 2009. p.98.

11. Rufián-Renares JA, Delgado-Andrade C, Morales FJ. Non-enzymatic browning: the case of the Maillard reaction. In: Delgado-Andrade C, RufiánRenares JA. Assessing the generation and bioactivity of neo-formed compounds in thermally treated foods. Granada: Atrio; 2009. p.9-32. 
12. Barbosa JHP, Oliveira SL, Seara LT. Produtos da glicação avançada dietéticos e as complicações crônicas do diabetes. Rev Nutr. 2009; 22(1):113-24. doi: org/10.1590/S1415-52732009000100011.

13. Vlassara $\mathrm{H}$. Advanced glycation in health and disease: role of the modern enviroment. Ann NY Acad Sci. 2005; 1043:452-60.

14. Monnier VM. Dietary advanced lipoxidation products as risk factors for human health: a call for data. Mol Nutr Food Res. 2007; 51:1091-3.

15. Nguyen CV. Toxicity of the AGE generated from the Maillard reaction: on the relationship of foodAGE and biological AGE. Mol Nutr Food Res. 2006; 50:1140-9.

16. Goldberg T, Cai W, Peppa M, Dardaine V, Baliga BS, Uribarri J, Vlassara H. Advanced glycoxidation end products in commonly consumed foods. J Am Diet Assoc. 2004; 104(8):1287-91.

17. Charissou A, Ait-Ameur L, Birlouez-Aragon I. Evaluation of gas chromatography/mass spectrometry method for the quantification of carboxymethyllysine in food samples. J Chromatogr. 2007; 1140:189-94.

18. Xanthis A, Hatzitolios A, Koliakos G, Tatola V. Advanced glycosylation end products and nutrition: a possible relation with diabetic atherosclerosis and how to prevent it. Food Sci. 2007; 72:125-9.

19. Nass N, Barling B, Santos NA, Scheubel RJ, Bögermann J, Silber RE, et al. Advanced glycation end products, diabetes and ageing. Z Gerontol. Geriatr. 2007; 40:349-56.

20. Uribarri J, Woodruff S, Goodman S, Cai W, Chen $X$, Pyzik R, et al. Advanced glycation end products in foods and a practical guide to their reduction in the diet. J Am Diet Assoc. 2010; 110:911-6.

21. Matiacevich SB, Buera MP. A critical evaluation of fluorescence as a potential marker for the Maillard reaction Silvia B. Food Chem. 2006; 95:423-30.

22. Föster $A$, Hühne $Y$, Henle T. Studies on absorption and elimination of dietary Maiillard reaction products. Ann NY Acad Sci. 2005; 1043:474-81.

23. Somoza V. Five years of research on health risks and benefits of Maillard reaction products: an update. Mol Nutr Food Res. 2005; 49:663-75.

24. Delgado-Andrade C, Seiquer I, Navarro MP, Morales FJ. Maillard reaction indicators in diets usually consumed by adolescent population. Mol Nutr Food Res. 2007; 51:341-51.

25. Delgado-Andrade C, Rufián Henares JA, Morales FJ. Lysine availability is diminished in commercial fibre-enriched breakfast cereals. Food Chem. 2007; 100:725-731.
26. Buetler T. Dicarbonyls in coffee. IMARS Highlights. 2008; 2(7):5-7.

27. Baynes JW. Dietary ALE are a risk to human health - NOT! Mol Nutr Food Res. 2007; 51:1102-6.

28. Seiquer I, Díaz-Alguacil J, Delgado-AndradeC, López-Frías M, Hoyos AM, Galdó G, et al. Diets rich in Maillard reaction products affect protein digestibility in adolescent male aged 11-14y. Am J Clin Nutr. 2006; 83:1082-8.

29. Ahmed N. Advanced glycation end-products: role in pathology of diabetic complications. Diabetes Res Clin Pract. 2005; 67(1):3-21.

30. Glenn JV, Stitt AW. The role of advanced glycation end products in retinal ageing and disease. Biochim Biophys Acta. 2009; 790:1109-16.

31. Tan KCB, Chow WS, Ai VHG, Metz C, Bucala R, Lam KSL. Advanced glycation end produtcs and endothelial disfunction in Type 2 diabetes. Diabetes Care. 2002; 25(6):1055-9.

32. Kim W, Hudson BI, Moser B, Guo J, Rong LL, Qu $W$, et al. Receptor for advanced glycation end products and its ligands: a journey from the complications of diabetes to its pathogenesis. Ann NY Acad Sci. 2005; 1043:553-61.

33. Sasaki N, Fukatsu K, Hayashi Y, Yoshida T, Fuji N, Koike $T$, et al. Advanced glyation end products in Alzheimer's diseases and other neurodegenerative diseases. Am J Pathol. 1998;153(4):1149-55.

34. Rojas A, Morales MA. Advanced glycation and endothelial functions: a link towards vascular complications in diabetes. Life Sci. 2004; 76:715-30.

35. Koschinsky T, He CJ, Mitsuhashi T, Bucala R, Liu C, Bueting $C$, et al. Orally absorbed reactive glycation products (glycotoxins): an environmental risk factor in diabetic nephropathy. Med Sci Res. 1997; 94: 6474-79.

36. Delgado-Andrade C, Rufiàn-Henares J, Morales FJ. Fast method to determine furosine in breakfast cereals by capillary zone electrophoresis. Eur Food Res Technol. 2005; 221:707-11.

37. Uribarri J, Cai W, Pappa M, Goodman S, Ferrucci L, Striker $\mathrm{G}$, et al. Circulating glycotoxins and dietary advancedglycation endproducts: two links to Inflammatory Response, oxidative stress and aging. J Gerontol. 2007; 62(4):427-33.

38. Rufiàn-Henares JA, Garcia-Villanova B, GuerraHernandez E. Determination of furfural compounds in enteral formula. J Liq Chromatogr. 2001; 24(19): 3049-61.

39. Uribarri J, Cai W, Sandu O, Peppa M, Goldberg T, Vlassara H. Diet-derived advanced glycation end 
904 | J. SHIBAO \& D.H.M. BASTOS

product are major contributors to the body's AGE pool and induce inflamation in health subjects. Ann NY Acad Sci. 2005; 1043:461-6.

40. Uribarri J, Peppa M, Cai W, Goldberg T, Lu M, He $C$, et al. Restriction of dietary glicotoxins reduces excessive advanced glycation end products in renal failure patients. J Am Soc Nephrol. 2003; 14(3): 728-31.

41. Teixidó E, Moyano E, Santos FJ, Galceran MT. Liquid chromatography multi-stage mass spectrometry for the analysis of 5-hydroxymethylfurfural in foods. J Chromatogr. 2008; 185:102-8.

42. Vlassara H, Palace MR. Glycoxidation: the menace of diabetes and aging. Mount Sinai J Med. 2003; 70(4):232-41.

43. Sandu O, Song KY, Cai WJ, Zeng, F, Uribarri, J, Vlassara, $H$. Insulin resistance and type 2 diabetes in high-fat-fed mice are linked to high glycotoxin intake. Diabetes. 2005; 54(8):2314-19.

44. Chun-Liang L, Chiu-Ching H, Chun-Chen Y, HuanYu Y, Feng-Rong C, Chih-Wei Y. Reduction of advanced glycation end product levels by on-line hemodiafiltration in long-term hemodialysis patients. Am J Kidney Dis. 2003; 42(3):524-31.

45. Rada-Mendoza M, García-Baños J, Villamiel M, Olanoa A. Study on nonenzimatic browning in cookies, brackers and breakfast cereals by maltulose and furosine determination. J Cereals Sci. 2004; 39:167-73.

Recebido em: 11/6/2010

Versão final reapresentada em: 21/6/2011 Aprovado em: 24/8/2011 